\title{
Impact of Production Location, Production System, and Variety on the Volatile Organic Compounds Fingerprints and Sensory Characteristics of Tomatoes
}

\author{
Mirthe Muilwijk, ${ }^{1}$ Samuel Heenan, ${ }^{1}$ Alex Koot, ${ }^{1}$ and Saskia M. van Ruth $^{1,2}$ \\ ${ }^{1}$ RIKILT Wageningen UR, P.O. Box 230, 6700 EV Wageningen, Netherlands \\ ${ }^{2}$ Food Quality and Design Group, Wageningen University, P.O. Box 17, 6700 AA Wageningen, Netherlands \\ Correspondence should be addressed to Saskia M. van Ruth; saskia.vanruth@wur.nl
}

Received 8 January 2015; Accepted 26 January 2015

Academic Editor: Manel Issaoui

Copyright (C) 2015 Mirthe Muilwijk et al. This is an open access article distributed under the Creative Commons Attribution License, which permits unrestricted use, distribution, and reproduction in any medium, provided the original work is properly cited.

\begin{abstract}
Consumers have more and more interest in where and how their foods are produced. However, it is often challenging to discriminate products from different production locations and systems. The objective of this study was to examine fingerprinting of volatile organic compounds (VOCs) as an approach for characterization and discrimination of tomatoes by their production location, production system, and variety using Proton Transfer Reaction Mass Spectrometry combined with multivariate statistics. Sensory analysis was complementing the VOC analyses. The study was part of the EU CORE Organic II project AuthenticFood. Tomato sample batches cultivated in two locations in Italy, according to the organic and conventional production system, comprising two varieties, and produced in two consecutive years were examined. Both factors production location and production system impacted considerably the VOC fingerprints, but compared to these two factors, minor differences were observed between the two varieties of tomatoes studied. VOC data were successfully used to predict the origin and production system for this sample set. Sensory data also primarily indicated the differences between origin and production systems, and several sensory attributes could be predicted from the VOC fingerprints. Therefore, VOC fingerprints reflect production conditions and are promising for substantiation and authentication of special tomato traits.
\end{abstract}

\section{Introduction}

In past years, the interest of consumers in the origin of their food has increased and more attention is paid to the location and the way of production of the food products purchased and consumed [1]. The way of production may relate to the organic production, fair trade, animal welfare considering practices, and so forth. The interest in organic foods has particularly increased over the last decades [2], which resulted in larger market shares [1]. The increased demand in combination with the higher prices compared to their regular counterparts make the specialty products susceptible to adulteration and counterfeiting. The existing certification system in the sector is pivotal to counteract these threats and would benefit from analytical methods allowing verification of the nature of the food products $[3,4]$. Various methods have been reported in the scientific literature for authentication of organic produce and products, for which we refer to the review of Capuano et al. (2013) [5]. However, in general there are fairly few analytical methods available to authenticate organic products which are thoroughly validated and ready to be used in practice. Furthermore, the scientific methods require often lengthy sample preparations and analyses. The current study is part of the EU project AuthenticFood. In the AuthenticFood project, novel analytical techniques for organic authentication are developed and tested. These are based on a suite of the most promising analytical methods such as multielemental analysis, stable isotope analysis, and metabolomics and pesticide screening. The project focuses on tomatoes and wheat as model plant materials. From the perspective of interesting metabolites and rapid measurements, the group of volatile organic compounds (VOCs) was 
selected as a group comprising relevant intrinsic markers for the identity and authenticity of tomatoes.

Over 400 VOCs have been detected in tomatoes, including hexanal, hexenal, methanol, ethanol, and acetaldehyde $[6,7]$. The various types of tomatoes can be distinguished from each other by the concentrations of the VOCs [79]. These are derived from three different precursors, to be known fatty acids, aromatic amino acids, and carotenoids [10]. In particular, the oxylipin pathway is an important one; in this pathway linoleic and linolenic acid are converted to $\mathrm{C6}$ compounds such as hexanal via lipoxygenase (LOX) [7]. The amino acids leucine, isoleucine, and phenylalanine are precursors for VOCs such as phenyl acetaldehyde and methyl salicylate [7]. In the carotenoid biosynthesis pathway, oxidative cleavage of carotenoids results in the presence of apocarotenoids such as $\beta$-ionine [7]. The stem and leaves of tomatoes contain VOCs as well; these VOCs are in particular terpenoids [8].

In previous studies on tomato characterization, sensory analysis has been applied as well. It was shown that volatiles originating from tomatoes are closely related to sensory descriptors [11, 12]. Although over 400 VOCs have been detected in tomatoes $[6,7]$, ca. 30 are considered as important contributors to fresh tomato flavor $[13,14]$.

For the measurement of VOCs in food, several analytical techniques are commonly used [15-20]. The technique most generally used for the analysis of VOCs in food is GC-MS $[7,10]$. However, this technique is fairly time consuming and expensive $[7,8,10]$. Other techniques that have been used previously concern sensor array systems (electronic nose or E-nose), headspace fingerprint mass spectrometry (HFMS), and Proton Transfer Reaction Mass Spectrometry (PTR-MS). The E-nose is designed to mimic the human perception and can be used for quality testing, but it is less suitable for authentication, as this system cannot detect specific VOCs. The HFMS is more suitable to use for authentication; its advantages are that it is not sensitive to water and alcohols and that it responds linearly to the concentration of VOCs. However, the HFMS leads to strong fragmentation what makes it a complex technique [7]. For measurements of VOCs for authentication of tomatoes, PTR-MS may be a suitable technique [21].

PTR-MS has been previously used to determine the aromatic profiles of tomatoes by monitoring the VOC emission [7, 22]. PTR-MS is a semistatic headspace technique, rapid, and highly sensitive and requires no pretreatment of samples [7]. After direct inlet of a mixture of VOCs, the compounds are protonized by $\mathrm{H}_{3} \mathrm{O}^{+}$. In general, fairly limited fragmentation occurs [21]. Concentrations can be measured as low as pg/g [23], and the response time per compound is in the order of seconds [21]. Therefore, this technique allows rapid analyses with high sensitivity. A drawback of the technique is that no distinction can be made for compounds with the same nominal mass [15].

The objective of the present study is to study the impact of production location (geographical origin), production system (organic or conventional), and tomato variety (Roma and San Marzano) on the VOC fingerprints using PTR-MS combined with multivariate statistics in view of characterization and authentication of the special traits of tomatoes. Furthermore, the fingerprints were used to substantiate and predict the sensory quality of the tomatoes.

\section{Experimental}

2.1. Sampling Procedure. Fresh tomato samples were delivered by partners of the EU project AuthenticFood. The set comprised of samples from two production locations in Italy (Basilicata in south Italy and Emilia Romagna in central Italy), two production systems (organic and conventional), two varieties (Roma and San Marzano), and two harvesting years (2012 and 2013). A full factorial $2 \times 2 \times 2 \times 2$ design was used, with for each category a duplicate sample batch and for each batch again triplicate sample analyses. The organic tomatoes were cultivated in agreement with the existing European legislation (EU Reg. 834/07). Tomato plants were planted at the end of April/beginning of May each year and the tomatoes were harvested in August/September of the same year. The soil in Basilicata consisted mostly of loamy soil, whereas the soil in Emilia Romagna consisted of clay. In the organic production, organic fertilizers and mechanical weed management were applied, and in the regular production a combination or organic and mineral fertilizers was used as well as chemical weed protection agents. Plant protection agents were applied in agreement with legislation requirements for organic and regular production of tomatoes. Harvesting quantities were 20-30 tons/ha in the organic production and 50-80 tons/ha in the regular production in Basilicata. In Emilia Romagna 50-60 tons/ha was harvested in the organic production and 70-90 tons/ha in the regular productions.

The tomato samples of 2012 were harvested at the redripe stage of maturity; however, some of the tomato samples deteriorated on route and therefore it was decided that the tomatoes of 2013 were harvested just before this optimum stage of maturity. Samples were stored and shipped in the dark at $4-8^{\circ} \mathrm{C}$ and were analyzed within three days after arrival in the laboratory in Netherlands.

2.2. VOC Fingerprinting by PTR-MS. Before analysis samples were allowed to acclimatize overnight at room temperature (approximately $21^{\circ} \mathrm{C}$ ). Whole tomatoes were sliced into $1 \mathrm{~cm}$ cubes and $6 \mathrm{~g}$ of each sample was placed into a $250 \mathrm{~mL}$ screw cap glass bottle. Prior to analysis by PTR-MS, bottles containing the samples were placed into a water bath for 30 minutes at $25^{\circ} \mathrm{C}$, allowing the headspace to form an equilibrium with the samples. After these 30 minutes of equilibration, the headspace was measured by a high sensitivity PTR-MS (Ionicon GmbH, Innsbruck, Austria).

The PTR-MS consists of a reaction drift tube and a quadruple mass spectrometer [21]. A standard procedure described by Ruiz-Samblás was used [24]. Briefly, the headspace is introduced into a drift tube at a flow rate of $48 \mathrm{~mL} / \mathrm{min}$. In an external hollow cathode ion, source which uses pure water vapor, $\mathrm{H}_{3} \mathrm{O}^{+}$ions are produced. In the drift 
tube, the volatiles are mixed with the $\mathrm{H}_{3} \mathrm{O}^{+}$ions, so that soft ionization of the VOCs with a higher proton affinity than water can take place. By a homogeneous electric field of $600 \mathrm{~V}$, the ionized volatiles are transported through the drift tube, whereafter the mass is analyzed in [ppbv] as their mass plus one (due to protonation), resulting in a volatile fingerprint [21]. The temperature of the inlet and drift chamber was kept at $60^{\circ} \mathrm{C}$. Cross-contamination between measurements was avoided by flushing the machine with clean air in between measurements and measurement of blank samples between all tomato sample measurements to monitor the background and allow for background subtraction. The full-scan mode was used and mass spectra were collected in a range of 20-160 atomic mass units (amu). A dwell time of $0.2 \mathrm{~s} /$ mass unit was used. For each replicate, a full mass scan was recorded. The data were background and transmission corrected, yielding one corrected mass spectrum per replicate. Then, the mass spectra of the three replicates of each sample were averaged to obtain a mean mass spectrum per batch sample. All resulted in 32 mean batch fingerprints and 96 individual fingerprints.

2.3. Sensory Analysis. The tomato samples of 2012 were assessed by a trained sensory expert panel consisting of 12 panelists who were selected and trained at the Greenhouse Horticulture Department of Wageningen UR, Netherlands. The panelists carried out quantitative descriptive analysis (QDA) and selected attributes were evaluated by scoring perceived intensities on a $0-100$ visual analogue scale [25]. Tomatoes were assessed in random order to avoid order effects.

2.4. Statistical Analysis. Principal Component Analysis (PCA) was performed on the VOC fingerprint data and the sensory data to screen the multivariate data for outliers and to explore the presence of any natural clustering in the data. PCA performs a reduction in the data dimensionality in order to facilitate the visualization of the multivariate data retaining as much as possible the information present in the original data. Subsequently, $k$-nearest neighbors algorithm $(k-\mathrm{NN})$ was used to develop a classification model for classification of the samples according to the various classes. $k$-NN is a nonparametric method and a sample is classified by a majority vote of its neighbors, with the sample being assigned to the class most common among its $k$-nearest neighbors. Sensory scores were predicted from the VOC fingerprint data using Principal Component Regression (PCR). PCR is a regression analysis technique that is based on PCA. It considers regressing the outcome (the scores of the sensory attributes) on a set of covariates (VOCs) based on a standard linear regression model, but it uses PCA for estimating the unknown regression coefficients in the model. Since data preprocessing can have a profound effect on the model results, several ways of data preprocessing were evaluated: none (raw data), autoscaling (scaling to unit variance), mean-centering, and log transformation. The optimal models were selected and their performance was examined. All statistical analyses were carried out using Pirouette 4.5 (Infometrix, Seattle, WA, USA).

\section{Results and Discussion}

3.1. VOC Fingerprinting by PTR-MS. All tomato samples were analyzed by PTR-MS. Two examples of VOC fingerprints are presented in Figure 1, that is, the mean VOC fingerprints for tomatoes originating from the Basilicata and Emilia Romagna regions.

A large variety and relatively high concentrations of VOCs were measured in the headspace of the tomatoes. The most abundant ions present were those with a mass-to-charge ratio of $\mathrm{m} / z$ 33, 34, 43, 45, 46, 47, 51, 55, 57, 59, 61, 69, 73, 75, 81, and 83. Similar results were reported by Farneti et al. 2012 [7], who studied the dynamics of VOC release from tomatoes upon artificial mastication measured by PTR-MS. Compared to that particular study, the predominant ions determined in our study would relate to the following compounds: $\mathrm{m} / \mathrm{z} 33$ (methanol), 43 (propanol), 45 (acetaldehyde), 47 (ethanol), 57 (pentanol or 1-butanol), 59 (acetone or propanal), 61 (acetic acid or ethyl acetate), 69 (pentanal or nonanal), 73 (butanal or 2-butanone), 81 (hexenal), 83 (hexanal), and 85 (n-hexanol or 1-penten-3-one) [7]. Additionally, measured ions $\mathrm{m} / \mathrm{z} 34$ and $\mathrm{m} / \mathrm{z} 46$ are naturally occurring isotopes of methanol and acetaldehyde which both are compounds predominantly present in the tomato VOCs profile.

The VOC fingerprints were examined using multivariate analysis which is particularly suitable for handling large data sets. PCA was conducted on the mean VOC data of the 32 samples. The data matrix consisted of 32 rows (means of samples) and 140 variables (masses). The first three dimensions of the PCA on the normalized and autoscaled data are presented in Figure 2. The figure presents the samples of the two production locations. It is obvious that the Basilicata samples cluster well (black symbols) and the samples from Emilia Romagna region show more variation (red symbols). The two samples scoring high on PC2 are those that showed some microbial deterioration due to the transport from Italy to Netherlands.

The relevance of the various masses for the PCA is presented in the modeling power plot in Figure 3. Very high modeling power $(>0.75)$ was exhibited by the ions with massto-charge ratio $m / z 47,48,53,55,56,67,68$, and 127 . $k$-NN models were developed for the auto-scaled normalized data for the samples of the two regions $(k=2)$. All 32 samples were classified correctly according to their production location.

As the PCA plot in Figure 2 shows, most variation was explained by the production location. However, in the higher dimensions, a separation according to production system (organic or conventional) was observed. $k$-NN models were developed using the autoscaled normalized VOC data $(k=$ 4). Prediction of the production system of the organic tomato samples resulted in a $100 \%$ success rate, whereas the identity of 15 out of the 16 conventional samples was predicted correctly (94\%). The misclassified sample was one of the 2012 samples. Prediction of the variety in a similar way was not very successful, as 9 out of the 32 samples were misclassified ( $72 \%$ successful), but variety can probably rather easily be determined by DNA methodology. Results indicate that the VOC fingerprints of the tomatoes in the sample set were 


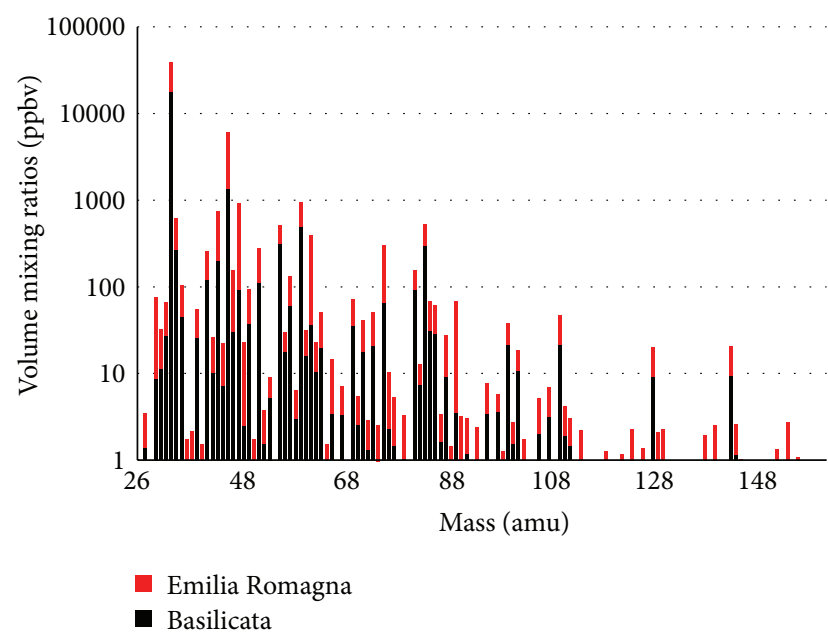

FIGURE 1: Mean VOC fingerprint of tomatoes originating from two regions measured by PTR-MS.

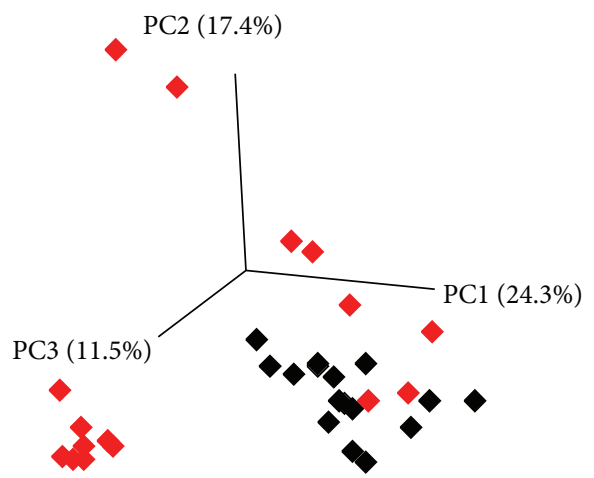

Figure 2: Plot of the first three dimensions of the Principal Component Analysis of the normalized and autoscaled VOC data of the 2012 and 2013 tomato samples from the Basilicata region (black symbols) and the Emilia Romagna region (red symbols).

predominantly affected by their location of production as well as by their production system.

\subsection{Sensory Analysis and Correlation with VOC Fingerprints.} The tomato samples of 2012 were subjected to additional sensory analysis. The results are presented in Table 1. For Basilicata samples, relatively high mean scores (score $>40$ ) were observed for the attributes acidity, pleasant aroma, being firm, fruit wall toughness, and being juicy.

The Emilia Romagna samples were characterized by high mean scores for the attributes acidity, aroma present, fruit wall toughness, being juicy, and being mealy. Organic samples presented relatively high scores for the attributes of being firm, juicy, aroma present, and aroma pleasant. Samples from regular production scored high on the same attributes but also on mealy and acidity. The two varieties showed both high scores for acidity, being firm, being juicy, aroma present, and aroma pleasant.

PCA analysis was carried out on the sensory data, the plot of which is presented in Figure 4. Like for the VOC analyses,

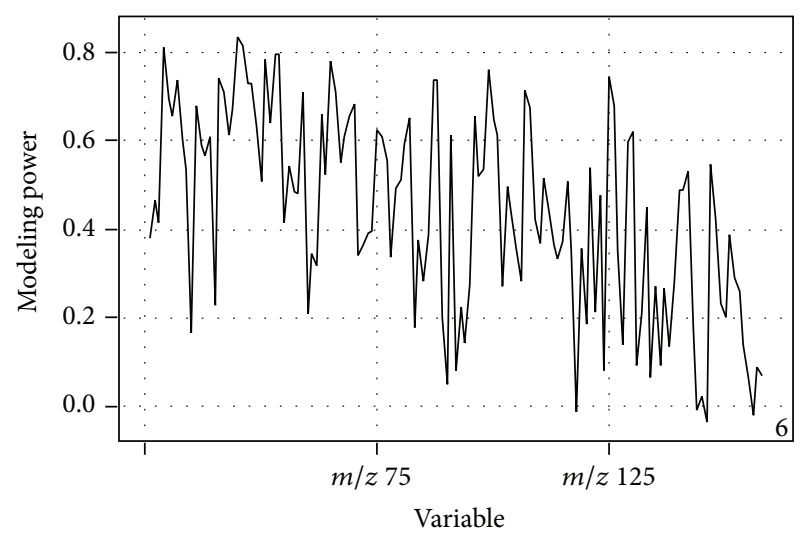

FIgURE 3: Modeling power of the individual variables (ions) in the Principal Component Analysis of the normalized and autoscaled VOC data of the 2012 and 2013 tomato samples from the Basilicata region and the Emilia Romagna region.

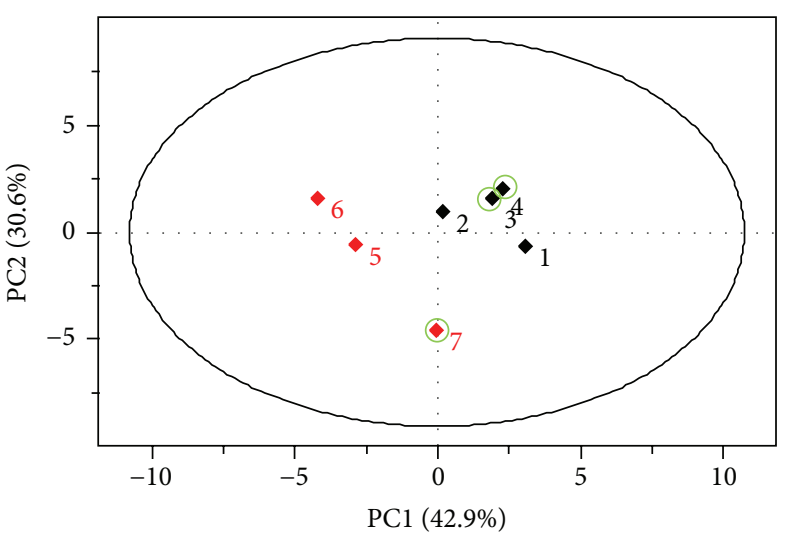

(a)

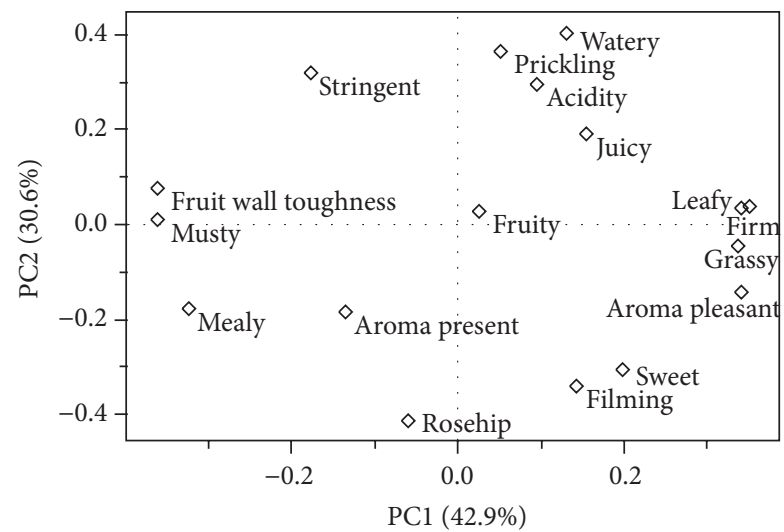

(b)

FIGURE 4: Scores (a) and loadings (b) plot of the first two dimensions of the Principal Component Analysis of the autoscaled sensory data of the 2012 tomato samples from the Basilicata region (black symbols) and the Emilia Romagna region (red symbols). Green circles in the scores plot indicate organic samples. 
TABLE 1: Results of sensory analysis of the 2012 tomato samples ${ }^{\mathrm{a}}$.

\begin{tabular}{|c|c|c|c|c|c|c|c|}
\hline & \multicolumn{7}{|c|}{ Samples } \\
\hline & 1 & 2 & 3 & 4 & 5 & 6 & 7 \\
\hline Production location & B & B & B & B & ER & ER & ER \\
\hline Production system & Reg & Reg & Org & Org & Reg & Reg & Org \\
\hline Variety & $\mathrm{R}$ & $\mathrm{SM}$ & $\mathrm{R}$ & SM & $\mathrm{R}$ & $\mathrm{SM}$ & $\mathrm{R}$ \\
\hline \multicolumn{8}{|l|}{ Attributes } \\
\hline Acidity & 49 & 50 & 43 & 40 & 40 & 41 & 27 \\
\hline Aroma pleasant & 51 & 38 & 43 & 46 & 33 & 30 & 46 \\
\hline Aroma present & 47 & 42 & 42 & 42 & 51 & 44 & 45 \\
\hline Filming & 8 & 7 & 7 & 6 & 7 & 6 & 8 \\
\hline Firm & 56 & 46 & 61 & 62 & 30 & 31 & 49 \\
\hline Fruit wall toughness & 45 & 54 & 51 & 51 & 69 & 76 & 53 \\
\hline Fruity & 17 & 16 & 17 & 15 & 18 & 15 & 15 \\
\hline Grassy & 15 & 14 & 13 & 12 & 8 & 7 & 12 \\
\hline Juicy & 56 & 44 & 53 & 56 & 52 & 49 & 44 \\
\hline Leafy & 11 & 9 & 10 & 13 & 7 & 3 & 8 \\
\hline Mealy & 25 & 41 & 23 & 25 & 47 & 50 & 48 \\
\hline Musty & 6 & 11 & 9 & 10 & 24 & 24 & 13 \\
\hline Prickling & 7 & 9 & 9 & 6 & 6 & 7 & 2 \\
\hline Rose hip & 9 & 8 & 6 & 5 & 9 & 7 & 12 \\
\hline Stringent & 6 & 8 & 8 & 9 & 7 & 11 & 6 \\
\hline Sweet & 39 & 29 & 34 & 40 & 30 & 28 & 48 \\
\hline Watery & 33 & 34 & 37 & 40 & 30 & 32 & 20 \\
\hline
\end{tabular}

${ }^{\mathrm{a}}$ Mean of QDA evaluations by 12 assessors; production location B = Basilicata; production location ER = Emilia Romagna; production system Reg $=$ regular production system; Org = organic production system; variety R = Roma; variety SM = San Marzano; the single sample from Emilia Romagna, from the organic production, and San Marzano variety that was analyzed by PTR-MS could not be assessed by the sensory panel due to safety concerns resulting from some microbial deterioration.

most variance was associated with the production origin, which is shown by the separation of the Basilicata and Emilia Romagna samples along the first PC. In the third dimension, the samples were separated according to the two production systems. No apparent separation according to variety was observed, which is consistent with the VOC results.

The VOC and sensory data sets presented in the previous sections were subsequently subjected to PCR in order to examine whether the VOC data would allow prediction of the sensory attribute scores. Examples of the sensory and VOC predicted scores for the musty attribute are presented in Figure 5. Over 20 masses contributed positively to the prediction and one mass contributed negatively; that is, a high sensory score was associated with a low intensity of the ion $m / z 49$.

The following Pearson correlation coefficients between actual and scores predicted from the autoscaled VOC data by PCR were obtained for the various attributes: acidity $(0.60)$, being aroma pleasant $(0.85)$, aroma present $(0.68)$, filming (0.55), being firm (0.95), fruit wall toughness (0.92), being fruity $(0.40)$, being grassy $(0.90)$, being juicy $(0.77)$, being leafy (0.76), being mealy (0.94), being musty (0.97), prickling $(0.70)$, rosehip $(0.80)$, being stringent $(0.51)$, being sweet $(0.7)$, and being watery $(0.84)$. The results indicate that VOCs relate to and are promising for the prediction of the intensity of several sensory attributes of the tomatoes

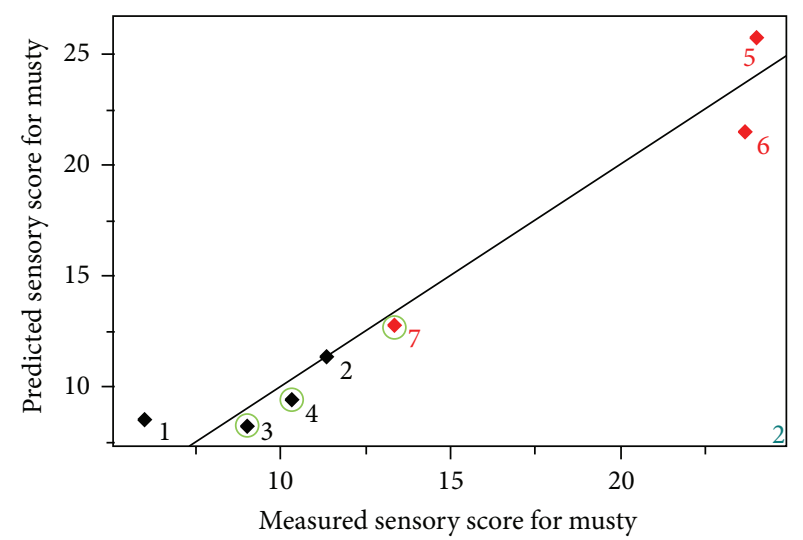

FIGURE 5: Comparison of measured sensory scores for musty and their predictions from the autoscaled VOC data set by Principal Component Regression of the 2012 tomato samples from the Basilicata region (black symbols) and the Emilia Romagna region (red symbols). Green circles in the plot indicate organic samples.

examined. Although the relationship between VOCs and sensory characteristics of tomatoes have been reported before $[9,12,13]$, the relation of both with production location and production systems for tomatoes is new. 


\section{Conclusions}

Tomato samples cultivated in two locations in Italy and according to the organic and conventional production system presented characteristic VOC fingerprints. Both factors production location and production system impacted considerably the concentrations of the VOCs, but compared to these two factors, minor differences were observed between the two varieties of tomatoes. VOC data were successfully used to predict the origin and production system for this sample set. Sensory data also indicated primarily VOC differences between origin and production systems, and several sensory attributes could be predicted from the VOC fingerprints.

\section{Conflict of Interests}

The authors of this paper report no conflict of interests and have no financial and personal relationships with other people or organizations that could influence their work.

\section{Acknowledgments}

The authors acknowledge the financial support provided by the CORE Organic II Funding Bodies, being partners of the FP7 ERANet Project, CORE Organic II (Coordination of European Transnational Research in Organic Food and Farming systems, Project no. 249667) via the AuthenticFood Project. Furthermore, the authors would like to acknowledge the funding of the research project from the Ministry of Economic Affairs in Netherlands, as well as through the Wageningen UR KB-project Authenticity-traceability. The authors gratefully acknowledge the EU CORE Organic II project partners for providing tomato sample material: Cristina Micheloni for the general coordination; Fabia Montalbano, Az. Agr. Vignola Giuseppe (Grassano, Italy), and Az. Agr. Masseria Bolettieri (Matera, Italy) for the samples from Basilicata; and Vincenzo Ritunnano, Coop. Dulcamara (Ozzano dell'Emilia, Italy), and Az. Agr. Contarini Alessandro (Codigoro, Italy) for the samples from Emilia Romagna. The authors also thank the sensory panel of the Greenhouse Horticulture Department of Wageningen UR for carrying out the sensory analyses.

\section{References}

[1] S. A. Drivelos and C. A. Georgiou, "Multi-element and multiisotope-ratio analysis to determine the geographical origin of foods in the European Union," TrAC-Trends in Analytical Chemistry, vol. 40, pp. 38-51, 2012.

[2] L. E. Ordóñez-Santos, M. L. Vázquez-Odériz, and M. A. Romero-Rodríguez, "Micronutrient contents in organic and conventional tomatoes (Solanum lycopersicum L.)," International Journal of Food Science \& Technology, vol. 46, no. 8, pp. 1561-1568, 2011.

[3] L. Nelson, J. Giles, C. MacIlwain, and V. Gewin, "Organic FAQ's," Nature, vol. 428, no. 6985, pp. 796-798, 2004.

[4] Y. Siderer, A. Maquet, and E. Anklam, "Need for research to support consumer confidence in the growing organic food market," Trends in Food Science and Technology, vol. 16, no. 8, pp. 332-343, 2005.

[5] E. Capuano, R. Boerrigter-Eenling, G. van der Veer, and S. M. van Ruth, "Analytical authentication of organic products: an overview of markers," Journal of the Science of Food and Agriculture, vol. 93, no. 1, pp. 12-28, 2013.

[6] H. J. Klee and D. M. Tieman, "Genetic challenges of flavor improvement in tomato," Trends in Genetics, vol. 29, no. 4, pp. 257-262, 2013.

[7] B. Farneti, S. M. Cristescu, G. Costa, F. J. M. Harren, and E. J. Woltering, "Rapid tomato volatile profiling by using protontransfer reaction mass spectrometry (PTR-MS)," Journal of Food Science, vol. 77, no. 5, pp. C551-C559, 2012.

[8] A. Z. Berna, J. Lammertryn, S. Saevels, C. Di Natale, and B. M. Nicolaï, "Electronic nose systems to study shelf life and cultivar effect on tomato aroma profile," Sensors and Actuators B: Chemical, vol. 97, no. 2-3, pp. 324-333, 2004.

[9] A. Z. Berna, S. Buysens, C. Di Natale, I. U. Grün, J. Lammertyn, and B. M. Nicolai, "Relating sensory analysis with electronic nose and headspace fingerprint MS for tomato aroma profiling," Postharvest Biology and Technology, vol. 36, no. 2, pp. 143-155, 2005.

[10] B. Farneti, A. A. Alarcón, S. M. Cristescu et al., "Aroma volatile release kinetics of tomato genotypes measured by PTR-MS following artificial chewing," Food Research International, vol. 54, no. 2, pp. 1579-1588, 2013.

[11] E. A. Baldwin, K. Goodner, A. Plotto, K. Pritchett, and M. Einstein, "Effect of volatiles and their concentration on perception of tomato descriptors," Journal of Food Science, vol. 69, no. 8, pp. S310-S318, 2004.

[12] K. S. Tandon, E. A. Baldwin, J. W. Scott, and R. L. Shewfelt, "Linking sensory descriptors to volatile and nonvolatile components of fresh tomato flavor," Journal of Food Science, vol. 68, no. 7, pp. 2366-2371, 2003.

[13] R. G. Buttery, R. Teranishi, R. A. Flath, and L. C. Ling, "Fresh tomato aroma volatiles: composition and sensory studies," in Flavor Chemistry: Trends and Developments, vol. 388 of ACS Symposium Series, pp. 213-222, American Chemical Society, Washington, DC, USA, 1989.

[14] R. G. Buttery and L. Ling, Bioactive Volatiles from Plants, ACS Symposium Series 525, American Chemical Society, Washington, DC, USA, 1993.

[15] A. Boschetti, F. Biasioli, M. van Opbergen et al., "PTR-MS real time monitoring of the emission of volatile organic compounds during postharvest aging of berryfruit," Postharvest Biology and Technology, vol. 17, no. 3, pp. 143-151, 1999.

[16] E. Boscaini, T. Mikoviny, A. Wisthaler, E. V. Hartungen, and T. D. Märk, "Characterization of wine with PTR-MS," International Journal of Mass Spectrometry, vol. 239, no. 2-3, pp. 215219, 2004.

[17] S. M. van Ruth, L. Dings, K. Buhr, and M. A. Posthumus, "In vitro and in vivo volatile flavour analysis of red kidney beans by proton transfer reaction-mass spectrometry," Food Research International, vol. 37, no. 8, pp. 785-791, 2004.

[18] S. M. van Ruth, L. Dings, E. Aprea, and S. Odake, "Comparison of volatile flavour profiles of kidney beans and soybeans by GCMS and PTR-MS," Food Science and Technology Research, vol. 11, no. 1, pp. 63-70, 2005.

[19] P. M. Granitto, F. Biasioli, E. Aprea et al., "Rapid and nondestructive identification of strawberry cultivars by direct PTRMS headspace analysis and data mining techniques," Sensors and Actuators, B: Chemical, vol. 121, no. 2, pp. 379-385, 2007. 
[20] E. Aprea, F. Biasioli, S. Carlin, I. Endrizzi, and F. Gasperi, "Investigation of volatile compounds in two raspberry cultivars by two headspace techniques: solid-phase microextraction/gas chromatography-mass spectrometry (SPME/GC-MS) and proton-transfer reaction-mass spectrometry (PTR-MS)," Journal of Agricultural and Food Chemistry, vol. 57, no. 10, pp. 4011-4018, 2009.

[21] D. Tholl, W. Boland, A. Hansel, F. Loreto, U. S. R. Röse, and J.-P. Schnitzler, "Practical approaches to plant volatile analysis," Plant Journal, vol. 45, no. 4, pp. 540-560, 2006.

[22] W. Lindinger, A. Hansel, and A. Jordan, "Proton-transferreaction mass spectrometry (PTR-MS): on-line monitoring of volatile organic compounds at pptv levels," Chemical Society Reviews, vol. 27, no. 5, pp. 347-354, 1998.

[23] M. Biniecka and S. Caroli, "Analytical methods for the quantification of volatile aromatic compounds," Track-Trends in Analytical Chemistry, vol. 30, no. 11, pp. 1756-1770, 2011.

[24] C. Ruiz-Samblás, A. Tres, A. Koot, S. M. van Ruth, A. GonzálezCasado, and L. Cuadros-Rodríguez, "Proton transfer reactionmass spectrometry volatile organic compound fingerprinting for monovarietal extra virgin olive oil identification," Food Chemistry, vol. 134, no. 1, pp. 589-596, 2012.

[25] H. T. Lawless and H. Heyman, Sensory Evaluation of Food Principles and Practices, Aspen Publishers, Baltimore, Md, USA, 2010. 

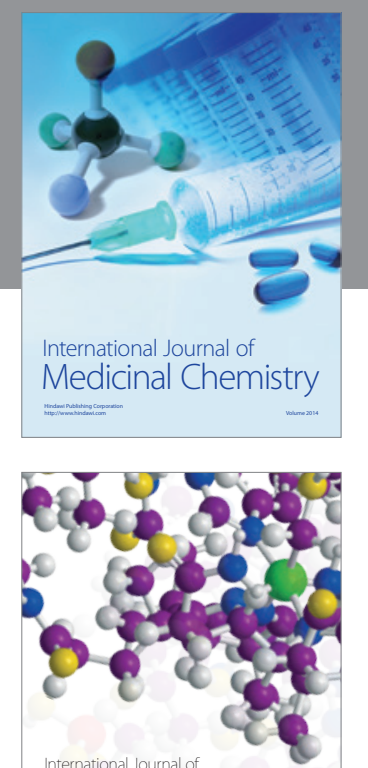

\section{Carbohydrate} Chemistry

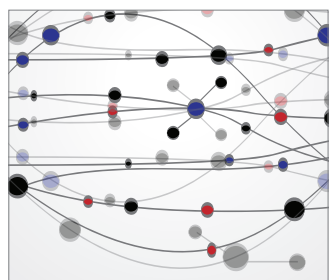

The Scientific World Journal
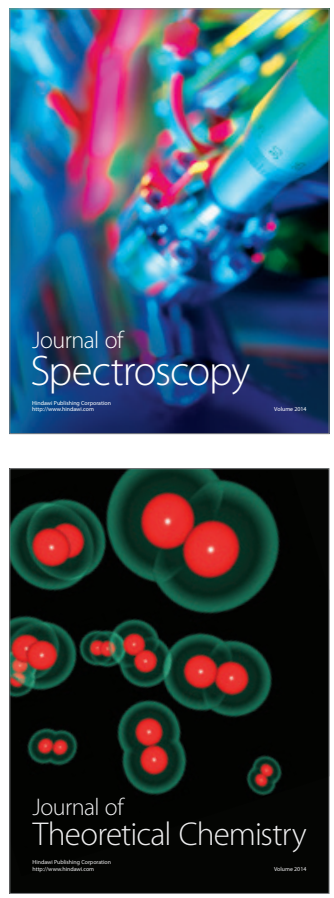
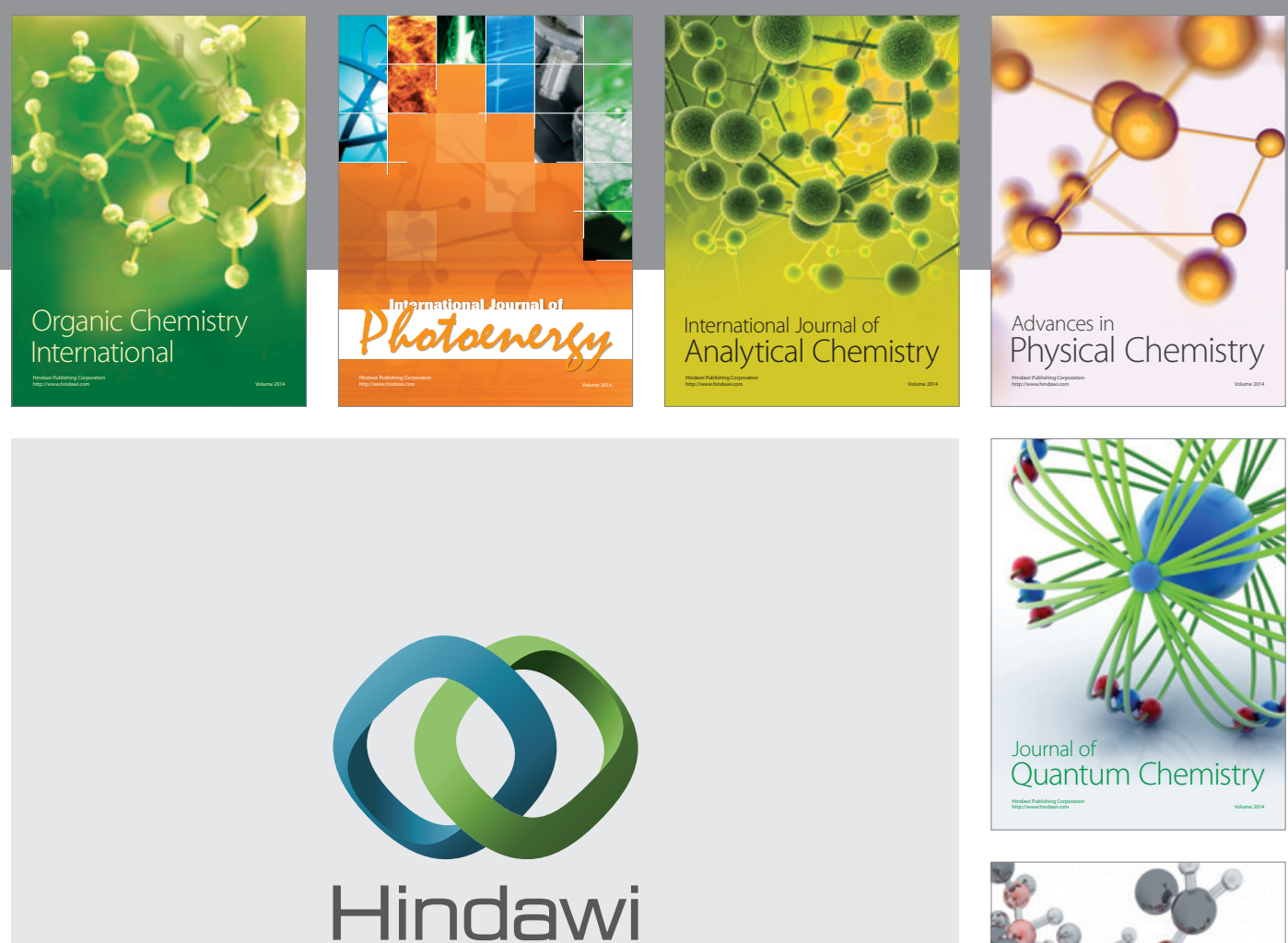

Submit your manuscripts at

http://www.hindawi.com

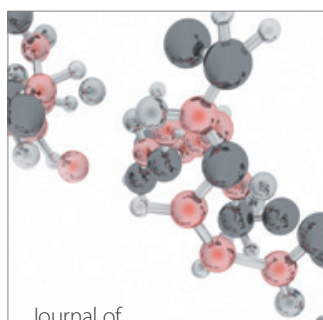

Analytical Methods

in Chemistry

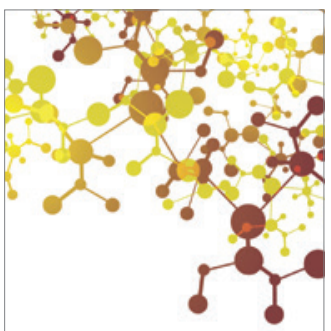

Journal of

Applied Chemistry

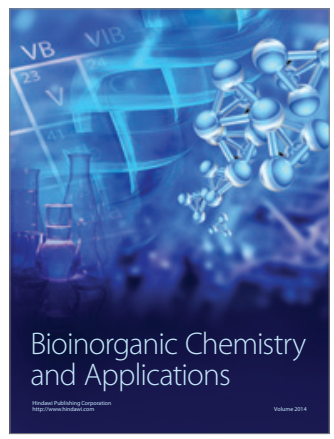

Inorganic Chemistry
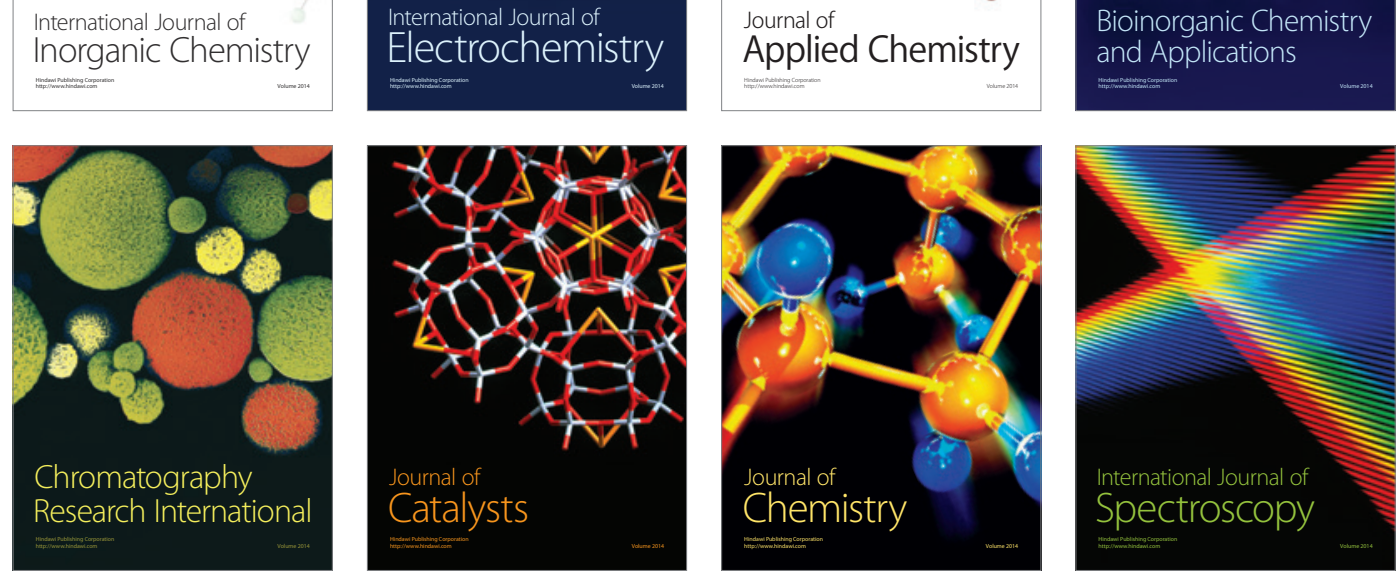